\title{
Head-mounted display for interactive inspection of painted free-form surfaces
}

\author{
Sören Kammel ${ }^{a}$ and Fernando Puente León ${ }^{b}$ \\ ${ }^{a}$ Institut für Mess- und Regelungstechnik, Universität Karlsruhe (TH), Karlsruhe, Germany \\ ${ }^{b}$ Lehrstuhl für Messsystem- und Sensortechnik, Technische Universität München, Germany
}

\begin{abstract}
Defects on painted surfaces have proven to be visually disturbing even when their depth is only a few microns. Most inspection approaches neither enable a reliable classification of small defects nor provide a suitable humanmachine interface to identify areas to be refinished. Consequently, in most cases the inspection still takes place manually and visually - an unsatisfactory compromise that lacks both objectivity and reproducibility. Our approach combines the reliability of automated methods with the acceptance and flexibility of human-based techniques. The measurement principle is based on deflectometry, and features a significantly higher sensitivity than triangulation methods. The developed system consists of a light source based on a digital micromirror device (DMD), a screen where defined patterns are projected on, as well as a mobile inspection device equipped with a head-mounted display (HMD) and a video camera. During operation, the camera captures images of different patterns reflected in the surface. By combining several images using one of the two techniques described to enhance surface defects, the resulting feature image is displayed in the HMD. This procedure takes place in real time and is repeated continuously. The system performance is demonstrated with the visual inspection of car doors. Promising results show that our prototype allows a reliable yet cost-efficient inspection of painted surfaces matching the needs of automotive industry.
\end{abstract}

Keywords: Automated visual inspection, head-mounted displays, image fusion, painted surfaces, car body parts, specular surfaces, deflectometry, structured illumination

\section{INTRODUCTION}

Painted free-form surfaces like car body parts play an important role in industrial production. A sufficiently accurate quality control is required to ensure that defects which are visually disturbing are detected and classified correctly even if they are only a few microns deep. During quality control of such surfaces, the detected defects often can be repaired within the final hand-finishing stage, provided they have been conveniently marked before. However, existing automated measurement systems for industrial painted surfaces that are able to classify and to mark even small defects reliably are expensive or do not provide a satisfactory human-machine interface enabling the inspector to identify the areas to be refinished:

- The sensitivity of triangulation methods (projection techniques, laser-light techniques, shadow techniques) and shape-from-shading techniques is not sufficient. In addition, these methods rely on the assumption of a diffusely reflecting surface. The specularity of painted surfaces complicates reliable measurements.

- Stylus instruments and optical autofocus scanners provide highly accurate measurements that allow a quantitative characterization of defects. However, these methods are too slow to be utilized in production lines. 1,2

- Techniques like laser or white light interferometry as well as Moiré methods are usually too sensitive to rough industrial environments or require a costly calibration. ${ }^{3,4}$

Further author information: (Send correspondence to S.K.)

S.K.: E-mail: kammel@mrt.uka.de, Telephone: +49 721608 3602, Address: Institut für Mess- und Regelungstechnik, Universität Karlsruhe (TH), Postfach 6980, D-76128 Karlsruhe, Germany, Internet: www.mrt.uni-karlsruhe.de

F.P.L.: E-mail: f.puente@ei.tum.de, Telephone: +49 89289 23347, Address: Lehrstuhl für Messsystem- und Sensortechnik, Technische Universität München, D-80290 München, Germany, Internet: www.emt.ei.tum.de 


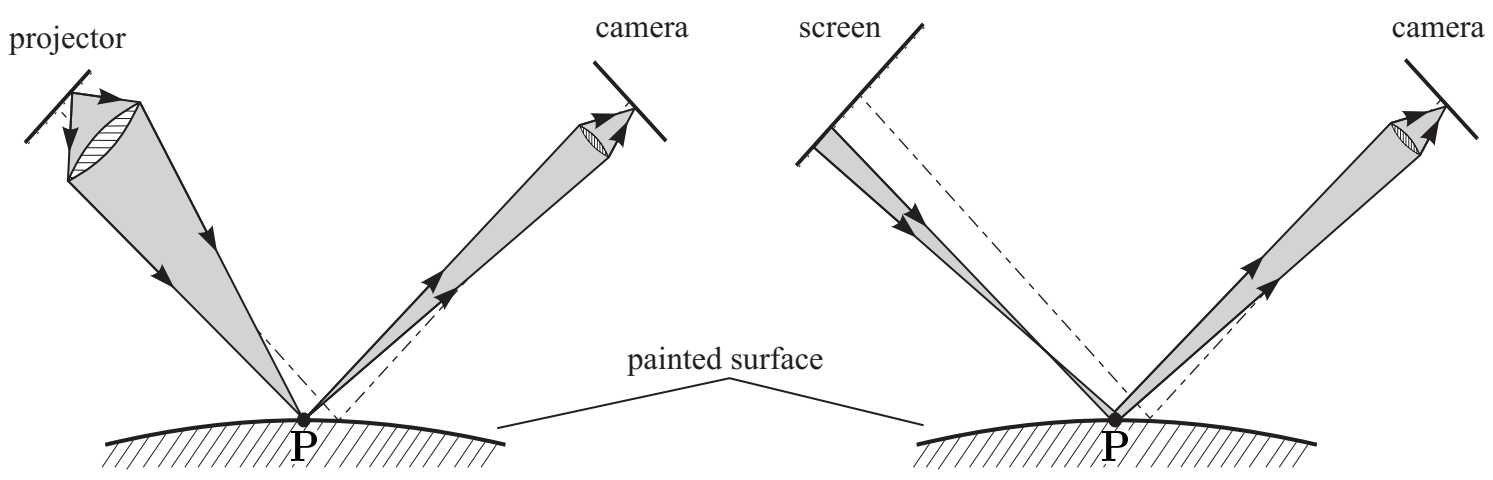

Figure 1. Measurement principles: (a) projection techniques; (b) deflectometry.

In contrast to these approaches, the two methods proposed in this paper combine a high sensitivity with the acceptance and flexibility of human-based inspection techniques. Both approaches are based on the deflectometric measuring principle. Basically, deflectometry reproduces the behavior of a human examiner, who observes and evaluates known patterns reflected in the surface being inspected, as described in Sect. 2.

Based on the deflectometric principle, a system for the interactive inspection of painted surfaces has been developed. It features a DMD-based light source projecting defined light patterns on a screen, as well as a mobile inspection device equipped with a head-mounted display (HMD) and a video camera. In the first stage, different patterns are generated, and images of their reflections in the surface are recorded by the camera. The acquired images are then combined using one of the methods described in Sect. 3. The processing of the images yields a feature image that is displayed in the HMD; see Sect. 4. This feature image provides an enhanced view of the surface showing defects like bumps, dents, streaks, bubbles or inclusions more clearly, as presented in Sect. 5 .

\section{DEFLECTOMETRIC INSPECTION}

Similarly to projection techniques, the deflectometric method discussed in this paper is also based on structured light patterns. Figure 1 compares their respective measurement principles.

When using a projection method like triangulation (Fig. 1(a)), the camera focusses on the surface, where a light pattern is projected on. Due to the parallax of the camera with respect to the projector, the pattern in the acquired image shows distortions compared to the original pattern. In contrast to that, Fig. 1(b) illustrates how images are formed when using deflectometric techniques. A camera focusses on a screen displaying a light pattern that is reflected in the surface.* This requires the surface to be at least partially specular. In this configuration, the surface becomes a part of the optical system and thus distorts the observed structured-light pattern.

The sensitivity of projection techniques and deflectometry to changes of the surface slope is compared in Fig. 2. If the surface is tilted at the point $\mathrm{P}$, the camera still observes the same point of the fringe pattern in case that a projection method is used; see Fig. 2(a). As shown in Fig. 2(b), the same change of the surface slope leads to mapping of a completely different area of the pattern on the screen when deflectometry is employed. In this measurement constellation, the light rays are deviated proportionally to the slope and to the distance between $\mathrm{P}$ and the structured-light pattern. Therefore, deflectometric techniques can be considered to be sensitive mainly to the surface slope. Since the surface is not observed directly, there is a trade-off between the lateral resolution and the sensitivity of the measurement system. This trade-off is balanced by the position of the focal plane of the camera. Focussing on the surface maximizes the lateral resolution of the surface being inspected at the expense of a reduced sensitivity. In contrast, focussing on the screen allows to exploit the highest resolution possible for the pattern, but also implies a blurring of the surface. Moreover, the sensitivity of the method can be enhanced by just increasing the distance between the surface and the screen.

${ }^{*}$ For a wide range of technical and engineering surfaces, the consideration of two-dimensional patterns has proven to be sufficient to perform the inspection. ${ }^{5}$ 


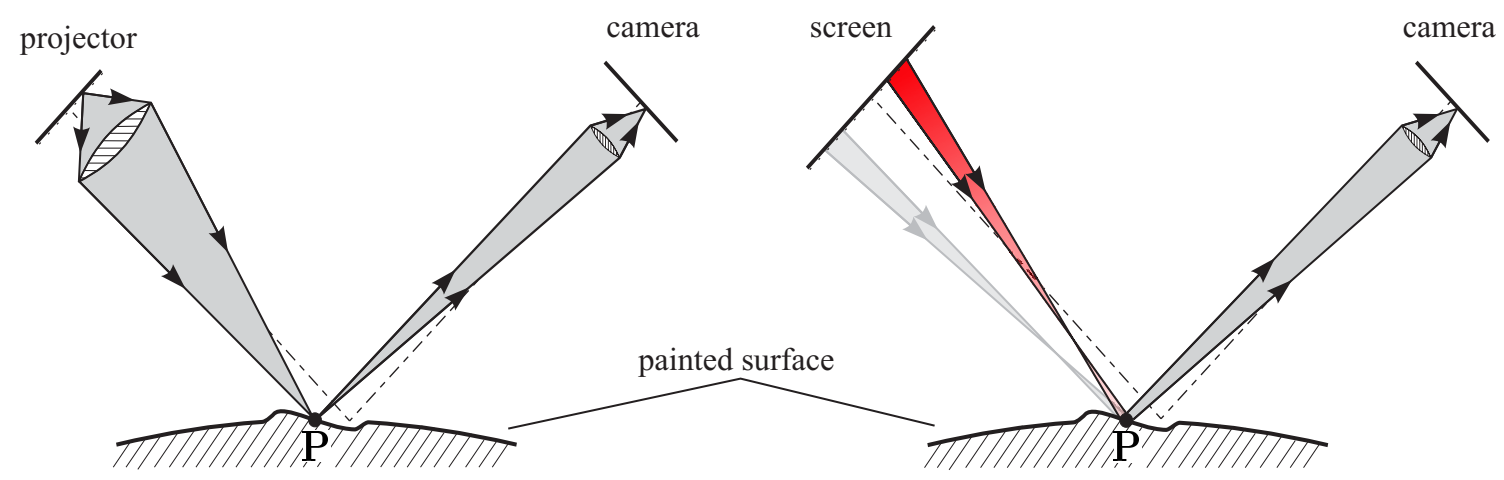

Figure 2. Comparison of the sensitivity to changes of the surface slope: (a) projection techniques; (b) deflectometry.

\section{SIGNAL ANALYSIS}

\subsection{Deflectometric Approach}

Deflectometry exploits the fact that at a specular surface, light is reflected according to the law of reflection. The camera observes the pattern displayed on the screen that is reflected by the surface, as shown in Fig. 1(b). To describe a scene consisting of a pattern, the painted surface under examination, and a camera, it is convenient to use more than one coordinate system. Points on the screen are described in the screen coordinate system $\left(\mathrm{O}^{\prime}, \mathbf{e}_{1}^{\prime}, \mathbf{e}_{2}^{\prime} ; \mathrm{SCS}\right)$, whereas points of the acquired images are given in the camera coordinate system $\left(\mathrm{O}, \mathbf{e}_{1}, \mathbf{e}_{2} ;\right.$ CCS); see Fig. 3. The origin $\mathrm{O}^{\prime}$ of the SCS is chosen to be the upper left corner of the screen. In general, the system is not calibrated and therefore the rigid body transform that maps the SCS to the CCS is not known. While the calibration is a prerequisite for shape measurement, it is not required for fast defect recognition.

The imaging function describes the relationship between the coordinates $\mathbf{l}=\left(l_{1}, l_{2}\right)^{\mathrm{T}}$ of a point $\mathrm{L}$ on the screen, described in the SCS, and the location $\mathbf{u}=\left(u_{1}, u_{2}\right)^{\mathrm{T}}$ of its mapping onto the camera sensor described in the CCS:

$$
\mathbf{l}\left(u_{1}, u_{2}\right)=\left(l_{1}\left(u_{1}, u_{2}\right), l_{2}\left(u_{1}, u_{2}\right)\right)^{\mathrm{T}} .
$$

For the sake of simplicity, patterns displayed on the screen are described in a Cartesian coordinate system $\left(l_{1}, l_{2}\right)$, but are actually projected onto the inside of a hemispherical screen to perform real measurements, as shown in Fig. 5(a). According to the law of reflection, a flat screen would restrict the deflectometric measurement system to the inspection of surfaces with a small spread of normal vector directions only. The hemispherical shape of the screen provides for visibility of the patterns on the screen after they have been reflected at the surface for the case of most free-form surfaces. ${ }^{6}$

Figure 3 illustrates the mapping described by the imaging function based on a simple pattern consisting of only one illuminated point. The imaging function for the point $\mathrm{L}_{0}$ of the pattern, whose coordinates $\mathbf{l}_{0}$ on the screen are assumed to be known, can be measured by searching the brightest point $\mathrm{U}_{0}$ in the acquired image and retrieving its coordinates $\mathbf{u}_{0}$.

To determine the imaging function for all sensor elements of the camera, the coordinates of the screen points can be coded to reduce the number of images to be acquired compared to the simple case described above. The coding of coordinates is a problem that is common to many measuring techniques, such as triangulation and shearography, and therefore a large number of coding schemes exists. ${ }^{7}$ Unfortunately, most of them are not usable for fast deflectometric measurements due to some peculiarities of this method:

- Binary codes like the Gray code require the camera to be focussed on the screen and need an adaptive control of the camera focus when used together with an HMD. Another disadvantage is that - even in the ideal case of a perfect optical mapping - only one-to-one correspondences between pixels on the screen and pixels on the camera sensor can be determined. Thus, high resolution patterns would be necessary to achieve a defect recognition with a reasonable spatial resolution. 


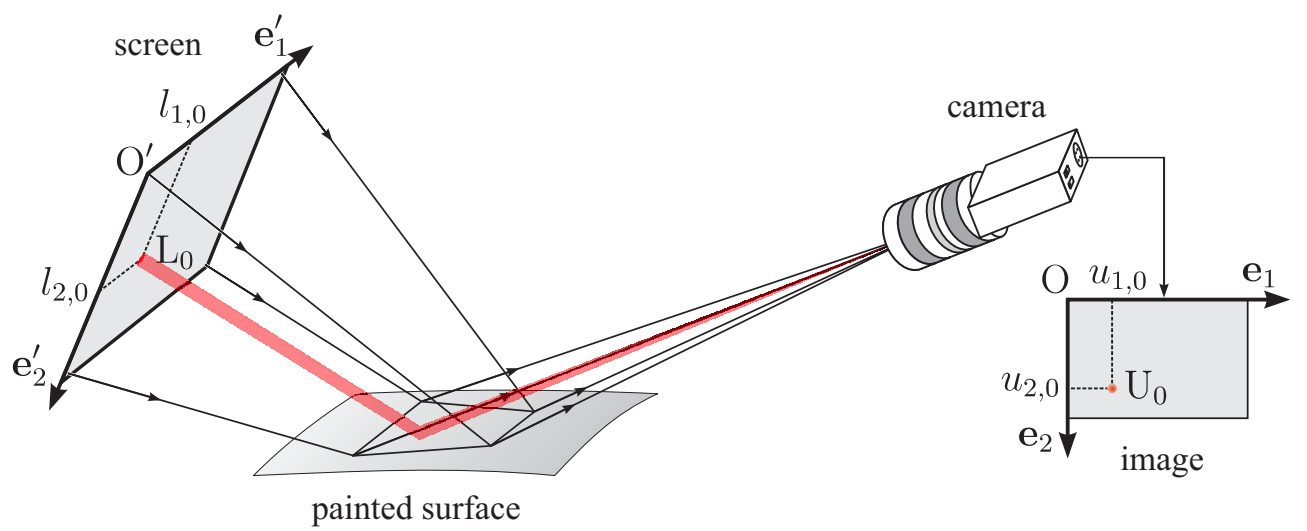

Figure 3. Measuring the imaging function for a single illuminated point.

- The surface to be inspected is a part of the optical system, and even areas with average curvature induce a distinct deformation of the reflected pattern. A one-dimensional DFT phase demodulation-a technique that requires a 1-D fringes with a known alignment of the reflected pattern - is therefore suited only for sufficiently flat surfaces and restricts the freedom of movement of the inspecting person. The twodimensional DFT phase demodulation suffers similarly from the large deformations of the reflected patterns. Though in principle, it is possible to determine the direction of the spectral components needed to retrieve the desired phase information, the automated design of a suited frequency filter is complicated and rather slow.

- Moiré techniques are based on the interference of two patterns. They require high carrier frequencies and are therefore sensitive to focal blur. Depending on whether the interference takes place optically or in a computer, two cases can be distinguished. Optical Moiré deflectometry requires an alignment to a reference grid or - if using the discrete structure of the camera sensor itself - a costly system calibration. In the case of numerical Moiré deflectometry, the signal analysis is similar to DFT phase demodulation. For this reason, the same restrictions apply to these two methods.

A class of coding techniques to which none of the restrictions mentioned above apply are phase shifting methods. With a carefully chosen decoding and phase unwrapping method, high quality measurement results can be achieved. For HMD-based applications, a fast measurement is important, and therefore as few phase steps as possible should be used. The minimum number of phase steps is offered by the well-known three bucket algorithm. On the other hand, this algorithm is susceptible to first order harmonics as are introduced by nonideal sinusoidal patterns. For deflectometric inspection, the reflection properties of the surface directly affect the quality of the images received by the camera. Therefore, it is hardly possible to prevent harmonic distortions of the patterns for every movement of the camera and for any kind of surface to be inspected. For this reason, the 4-bucket phase decoding algorithm is used that tolerates first order harmonics and has proven to process deflectometric data reliably.

However, phase shifting algorithms have an important drawback. They deliver values limited to the range of one period of the used sinusoidal patterns. Thus, a phase unwrapping algorithm has to be employed. Algorithms that unwrap the phase information without further measurements usually assume a smooth surface. The phase ambiguities caused by discontinuities and noise cannot be resolved in every case. For the usage together with an HMD, where no assumptions on the measurement constellation can be made, these algorithms are unsuited. Therefore, the phase unwrapping is performed with information obtained from additional measurements. To retain the advantageous properties of the phase shifting method, a second phase image is calculated using a wavelength different to the one used during the first measurement. Unwrapping the phase from the two wrapped phase images using the chinese remainder theorem (Nonius principle) produced unsatisfactory results, because 
the underlying integer arithmetic requires low-noise images as well as nearly ideal sinusoidal patterns. Such patterns, however, are usually not achievable with conventional optical devices.

A more robust approach is to iteratively refine the phase values starting with a coarse period, and to reduce the period of the sinusoidal patterns until the desired measurement accuracy has been reached. The starting period $q_{1}$ is chosen large enough to cover the whole measuring range, and thus it delivers a very coarse estimate of the phase values without phase jumps. Then, a second phase image is calculated using a fraction of the starting period length and is subsequently unwrapped using the first phase image. The unwrapping is done by adding $n(\mathbf{u}) \cdot q_{2}, n(\mathbf{u}) \in \mathbb{Z}$, to each point of the second image to ensure that the difference between both phase images does not exceed the period length $q_{2}$ of the second image at any point. To increase the speed of the unwrapping process and to avoid distortions that may confuse the user of the HMD, regions with low modulation are masked. As a measure of the modulation, the spread of each pixel in the coarse phase image series is used.

The resulting phase image contains the values of the imaging function according to Eq. (1), which are proportional to the local gradient of the surface. Due to the high dynamic range of the phase image - which actually represents coordinate values in the SCS-, the imaging function has to be processed for the purpose of defect visualization. First, the magnitude of the gradient of the imaging function is calculated using a simple discrete difference operator. Differentiating the values of the imaging function results in values proportional to the local surface curvature and therefore highlights changes of the surface slope. Subsequently, the image is inverted to stress the dynamic range of small slope deviations. Prior to the inversion, the constant one is added to avoid divisions by zero. The inverted data is basically proportional to the radius of the local curvature, and is displayed on the HMD for the purpose of surface inspection. A summary of the deflectometric measuring approach is depicted in Fig. 4.

\subsection{Image Fusion Approach}

In the scope of this paper, fusion is concerned with the combination of $B$ images

$$
\mathcal{D}:=\left\{d\left(\mathbf{u}, p_{i}(\mathbf{l})\right), i=0, \ldots, B-1\right\}
$$

to the desired result $r(\mathbf{u})$, where $\mathcal{P}=\left\{p_{i}(\mathbf{l}), i=0, \ldots, B-1\right\}$ represents the set of patterns displayed on the screen, and the magnitude $p_{i}(\mathbf{l})$ describes the intensity of the pattern at the location indicated by the vector $\mathbf{l}$. Although in the examples presented in Sect. 5 the result $r(\mathbf{u})$ is a scalar feature image, in other applications one could be interested in obtaining images, symbolic image descriptors, or even a vector containing several results instead; see Ref. 8.

The fusion of the sensor data shall take place complementarily. Though the information of interest is distributed over all the images of the series, for a certain location it remains concentrated to a few images. With regards to the fusion strategy to be employed, a centralized approach provides the most robust way to accomplish this task. Since the information fused is closer to the source, a better exploitation of the raw data is achieved. ${ }^{9}$ A disadvantage of such methods is that the resulting algorithms are often ad hoc solutions which in addition tend to be computationally expensive. Thanks to the homogeneity of the sensor data, however, a preprocessing is not needed in the present case. Thus, the computing time can be kept within a reasonable limit.

\subsubsection{Energy Minimization Strategy}

A fairly general approach to centralized data fusion is to express all knowledge available a priori on desirable properties and reasonable constraints regarding the raw data $\mathcal{D}$, the nuisance parameters ${ }^{10} \mathcal{N}$, and the fusion results $\mathcal{R}$ as well as their interconnections in shape of generalized energy terms $E_{k}(\mathcal{D}, \mathcal{R}, \mathcal{N}) .{ }^{11}$ 


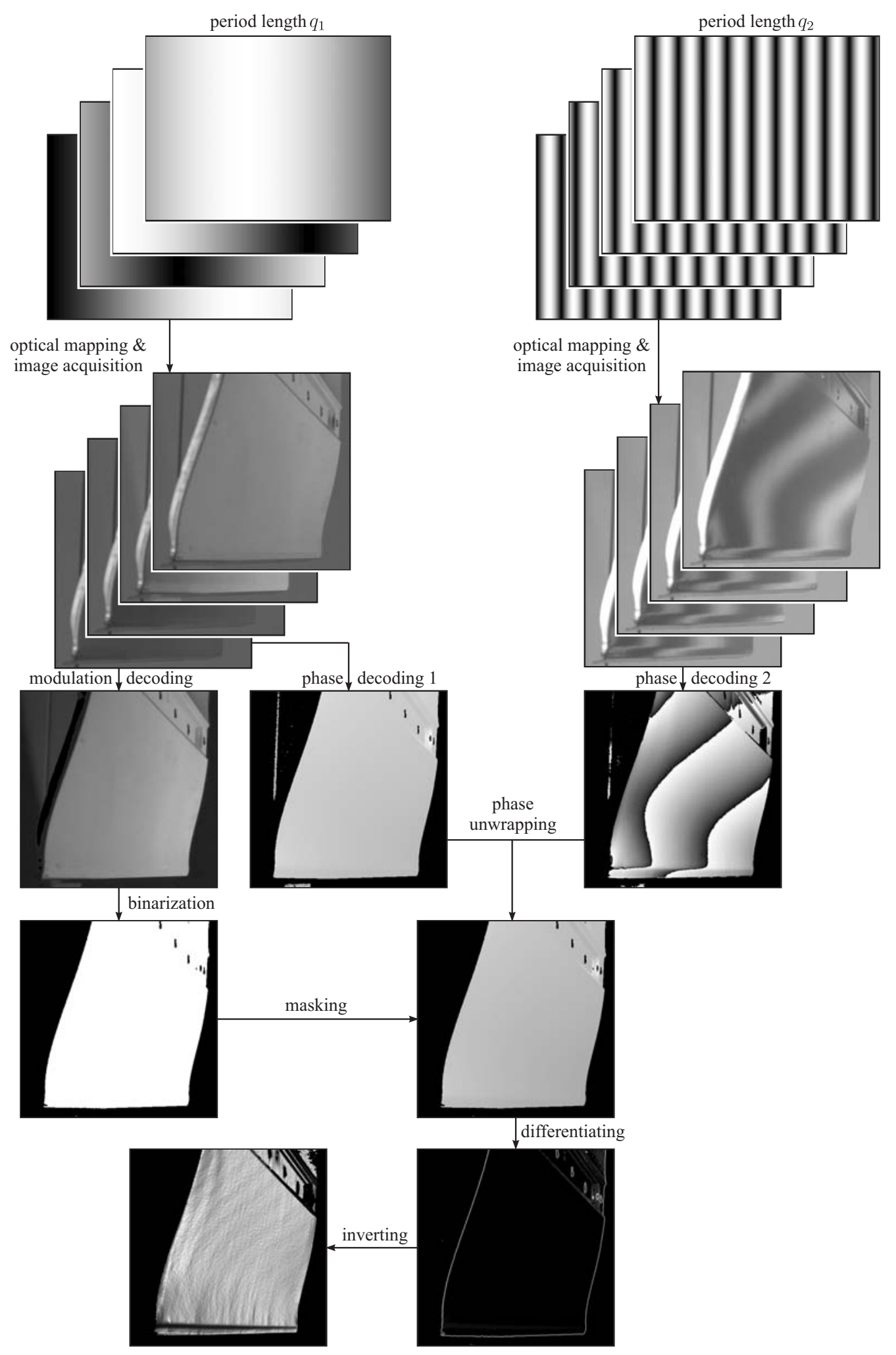

Figure 4. Scheme of the deflectometric defect detection approach. 
These "energies" $E_{k}$ have to be chosen such that the knowledge available and the requirements expressed are reflected monotonically in the sense that the result becomes more desirable the lower the energy is. The energy terms are then combined to an energy function $E$ by means of weighted summation:

$$
E=\sum_{k} \lambda_{k} E_{k}(\mathcal{D}, \mathcal{R}, \mathcal{N}), \quad \lambda_{k}>0
$$

$E$ represents an implicit approach to the fusion task. Due to the monotony of the energy function $E$, the fusion can be accomplished by minimizing it with respect to the fusion results $\mathcal{R}$ and the nuisance parameters $\mathcal{N}$ :

$$
\left\{\mathcal{R}^{*}, \mathcal{N}^{*}\right\}=\arg \min _{\mathcal{R}, \mathcal{N}}\{E\}
$$

where $\mathcal{R}^{*}$ and $\mathcal{N}^{*}$ denote the results of the optimization.

Advantages of this approach are its generality as well as the possibility to incorporate additional information and constraints by simply adding further energy terms. An important drawback, however, is the potential difficulty to optimize complex energy functions. ${ }^{8}$

\subsubsection{Fusion Algorithm}

To perform a reliable inspection, an image series $\mathcal{D}$ of the surface is recorded using a set of two-dimensional binary patterns generated according to the following equation:

$$
p_{i}(\mathbf{l})=\frac{1}{2}\left[\operatorname{sgn}\left(\cos \left(n \cdot \frac{2 \pi l_{1}}{l_{1, \max }}-\frac{2 \pi i}{B}\right)\right)+1\right],
$$

where $\operatorname{sgn}($.$) denotes the signum function:$

$$
\operatorname{sgn}(x)=\left\{\begin{array}{ll}
1 & \text { for } x \geq 0 \\
-1 & \text { otherwise }
\end{array},\right.
$$

In this equation, $l_{1, \max }$ denotes the maximum coordinate value in $l_{1}$ direction displayable by the device generating the patterns (e.g. the projector), and $n$ is the number of periods to display. The course of the image intensities at a fixed location $\mathbf{u}$ describes a typical signal pattern that enables one to recognize defective regions by means of appropriate analysis techniques.

In addition to defects like scratches, which cause local changes of the reflectance properties of the surface, bubbles and inclusions in the paint or coating should be detected as well. These defects are characterized by a much higher curvature as compared with intact surface regions. Therefore, from defective surface areas, the camera receives a compressed view of a large region of the screen. Details of the screen cannot be discriminated from pixels of defective areas, because of the discrete nature of the image formation as well as out-of-focus imaging. The defects are imaged with the mean brightness of the viewed screen area.

In contrast, the parts of an image that the camera receives from faultless regions ideally show a sharp image of the pattern on the screen. To ensure sharp images with high contrast in non-defective areas, it is recommendable to adapt the displayed pattern according to the curvature of the surface to examine, if possible. ${ }^{12}$ Displaying consecutive phase shifted patterns $p_{i}(\mathbf{l})$ results in distinct intensity changes within the areas of the camera image that correspond to faultless surface regions. Defective regions, however, show nearly the same constant grey level. This means, the contrast and therefore also the spread of any point $\mathbf{u}$ in the dimension spanned by the index $i$ denoting the phase of the patterns is high for faultless surface areas and low for defective regions. A suitable measure for the contrast is defined as:

$$
m_{\mathrm{c}}(\mathbf{u}):=\max _{i}\left\{d\left(\mathbf{u}, p_{i}\right)\right\}-\min _{i}\left\{d\left(\mathbf{u}, p_{i}\right)\right\} \quad \text { with } \quad i=0, \ldots, B-1 .
$$

The measure $m_{\mathrm{c}}(\mathbf{u})$ performs well, if the surface to be inspected shows a high portion of specularity, and the patterns used have high contrast. On the other hand, it is rather susceptible to noise due to its dependency on the extremal intensities. Thus, for noisy images it is preferable to use other measures, such as the variance

$$
m_{\mathrm{v}}(\mathbf{u}):=\frac{1}{B-1} \sum_{i=0}^{B-1}\left(d\left(\mathbf{u}, p_{i}\right)-\bar{d}(\mathbf{u})\right)^{2} \quad \text { with } \quad \bar{d}(\mathbf{u})=\frac{1}{B} \sum_{i=0}^{B-1} d\left(\mathbf{u}, p_{i}\right) .
$$



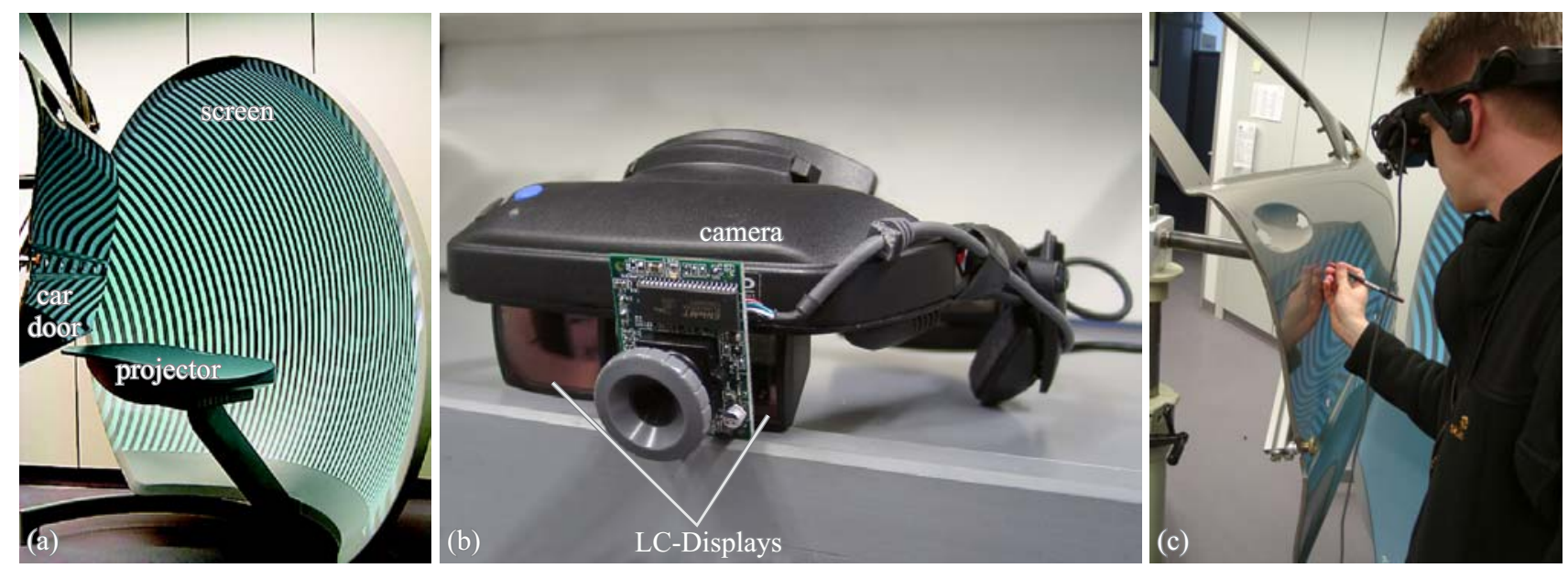

Figure 5. Inspection devices: (a) inspection environment; (b) HMD; (c) HMD-based interactive inspection of a car door.

The resulting energy function is trivial and consists of only one addend:

$$
E=\sum_{\mathbf{u}}[r(\mathbf{u})-m(\mathbf{u})]^{2}=E_{\text {feature }}(m(\mathbf{u}), r(\mathbf{u})) .
$$

Since for the optimal solution $r^{*}(\mathbf{u})=m(\mathbf{u})$ holds, a costly minimization is not necessary in this case.

\section{HMD-BASED INSPECTION SYSTEM}

A major requisite for a robust in-line inspection of painted surfaces is to combine the reliability of automated systems with the flexibility of human-based techniques to enable an efficient manual refinishing of defective areas. Stepping up to this challenge, we have developed a system that consists of a programmable, DMD-based light source, an arched screen, as well as a mobile inspection device equipped with a head-mounted display (HMD) and a video camera, as shown in Figs. 5(a) and (b). The screen has a diameter of $1.5 \mathrm{~m}$. Arbitrary patterns can be projected onto it by using a special fish-eye lens placed in its focal point. The HMD consists of two LC-Displays to visualize the feature image, as well as a camera used to record the raw data. In Fig. 5(c), operation of our system is demonstrated based on the inspection of a car door.

During operation, the camera continuously captures images of different fringe patterns reflected in the painted surface, thus emulating the behavior of human examiners. However, before displaying the images in the HMD, they are combined by means of the signal analysis techniques described in Sect. 3 to form an image in which defects appear efficiently enhanced. This way, the information gathered from multiple lighting constellations is concentrated in one single image. Additionally, quantitative detection results can be overlaid to the visualized image to provide for an objective assessment.

\section{RESULTS}

To demonstrate the performance of the signal processing algorithms implemented in our HMD-based system in the context of inspection of car body parts, the measurement setup shown in Fig. 5 has been used. The car body part, in this case a painted car door, has been placed above the projector.

Figure 6(a) shows an image of an area of the car door reflecting a binary fringe pattern. It is difficult to recognize any defects in this image. In Fig. 6(b), however, the phase measuring deflectometric approach according to Subsect. 3.1 has been applied to a series of images acquired using sinusoidal fringe patterns. The resulting feature image shows even small curvature deviations with a high spatial resolution. The high sensitivity allows to visualize deformations caused by a misadjustment of the forming machine that are nearly invisible to a human observer. Note that in this case the depth of the defects visualized are in the order of magnitude of only a few microns! Small bubbles and inclusions, however, cannot be recognized in this image. 
In Fig. 6(c), the fusion strategy described in Subsect. 3.2 has been applied to a series of images taken with the camera focussing on the screen. This focus setting maximizes the sensitivity of the system at the cost of low spatial resolution. Regarding the sensitivity, this method is comparable to the deflectometric approach.

In contrast, Fig. 6(d) shows the result of fusing images taken with the camera focussing on the door region directly. With this setting, the resolution is maximized at the expense of a reduced sensitivity. The increased resolution allows a reliable detection of paint defects such as bubbles, inclusions or scratches with a magnitude hardly measurable with alternative methods.

Obviously, since the different focus settings allow to image different spatial frequency components of the surface being inspected, both fusion results presented in Figs. 6(c) and (d) complement each other. Together, they provide a much more accurate insight into surface defects as compared with alternative techniques to inspect painted surfaces.

\section{CONCLUSIONS}

Two methods for an HMD based inspection of painted surfaces have been presented. The first one is based on a phase measuring deflectometric approach, whereas the other one is based on a novel centralized fusion technique. Both methods feature a high sensitivity with respect to small deviations of the surface gradient, and they allow a fast and clear visualization of regions that need to be refinished manually.

The first method relies on phase measuring deflectometry and yields quantitative results regarding curvature deviations. Using this technique, even small bumps and dents can be visualized with a high spatial resolution.

The second approach is based on image fusion techniques, and features a very robust behavior. To record the different images to be fused, complementary imaging conditions are systematically generated by projecting binary fringe patterns on a screen. To accomplish the fusion, a powerful centralized approach based on energy minimization has been selected. With the proposed fusion technique, not only scratches, bubbles and inclusions can be detected reliably. Moreover, with the very same setup, also bumps and dents can be visualized just by shifting the focal plane of the camera used.

The range of applications of the described methods can easily be extended to diffusely reflecting surfaces, such as unpainted car body parts, by using light of a larger wavelength and recording the corresponding images with an infrared camera.

In both cases, the results show that a reliable yet cost-efficient inspection of painted surfaces is attained that matches the needs of industry. In contrast to existing inspection approaches, the expense regarding the acquisition of the image data as well as the marking of defective areas has been significantly reduced thanks to an improved signal analysis and the use of an HMD.

\section{ACKNOWLEDGMENTS}

The authors would like to thank Mr. Michael Heizmann for valuable comments on a draft version of this paper.

\section{REFERENCES}

1. D. J. Whitehouse, Handbook of Surface Metrology, Institute of Physics Publishing, Bristol, 1994.

2. P. M. Lonardo, D. A. Lucca, L. De Chiffre, "Emerging trends in surface metrology," CIRP Annals 51/2, 2002.

3. T. Dresel, G. Häusler, H. Venzke, "3D-sensing with a confocal microscope," Applied Optics 31, 919-925, 1992.

4. C. G. Masi, "Moiré interferometry spots windshield defects," Image Processing Europe 5 (6), 22-25, 2002.

5. F. Puente León and S. Kammel, "Image fusion techniques for robust inspection of specular surfaces," in: Multisensor, Multisource Information Fusion: Architectures, Algorithms, and Applications VII, B. V. Dasarathy, ed., Proc. SPIE 5099, 2003.

6. J. Beyerer and D. Pérard, "Automatische Inspektion spiegelnder Freiformflächen anhand von Rasterreflexionen," Technisches Messen 64 (10), pp. 394-400, 1997. 

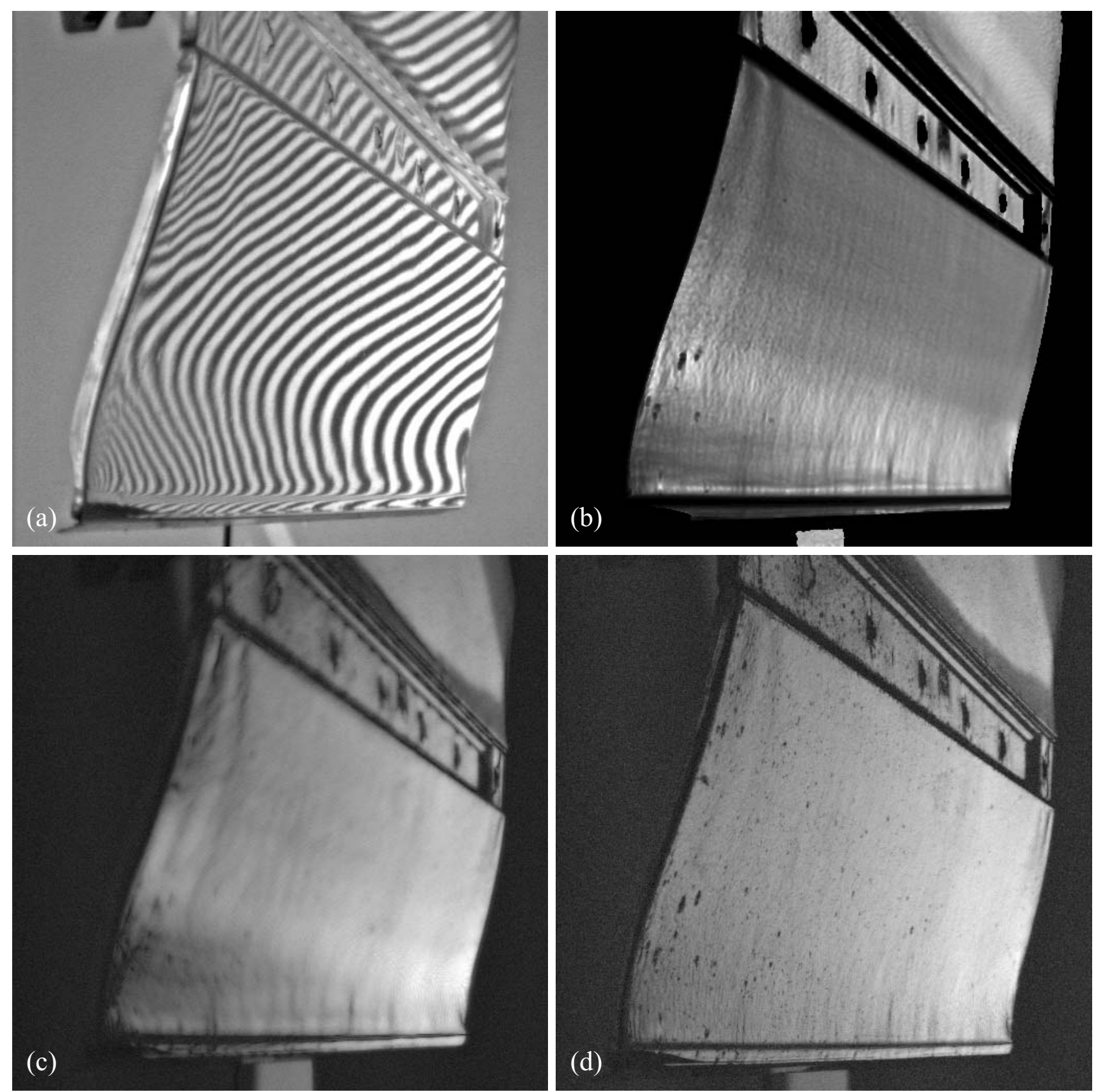

Figure 6. Results of the signal analysis: (a) image of an area of a car door reflecting a binary fringe pattern; (b) resulting feature image obtained with the deflectometric approach; (c) fusion result for the image series recorded while focussing on the screen; (d) fusion result for the image series recorded while focussing on the door.

7. D. Pérard, Automated visual inspection of specular surfaces with structured-lighting reflection techniques, Fortschritt-Berichte VDI, Reihe 8, No. 869, VDI Verlag, Düsseldorf, 2001.

8. F. Puente León, "Complementary image fusion for inspection of technical surfaces" (in German), Technisches Messen 69 (4), pp. 161-168, 2002.

9. D. L. Hall, Mathematical Techniques in Multisensor Data Fusion, Artech House Inc., Norwood MA, 1992.

10. J. Beyerer, "Is it useful to know a nuisance parameter?," Signal Processing 68 (1), pp. 107-111, 1998. 
11. J. J. Clark und A. L. Yuille, Data Fusion for Sensory Information Processing Systems, Kluwer Academic Publishers, Boston, 1990.

12. S. Kammel, "Automated optimization of measurement setups for the inspection of specular surfaces," in: Machine Vision and Three-Dimensional Imaging Systems for Inspection and Metrology II, K. G. Harding and J. W. V. Miller, eds., Proc. SPIE 4567, pp. 199-206, 2002.

13. F. E. Nicodemus, J. C. Richmond, J. J. Hsia, I. W. Ginsberg, and T. Limperis, Geometrical Considerations and Nomenclature for Reflectance, NBS Monograph 160, National Bureau of Standards, U.S. Department of Commerce, Washington DC, 1977.

14. G. Farin, Curves and Surfaces for Computer Aided Geometric Design, Academic Press, London, 1997.

15. P. Kierkegaard, "Reflection properties of machined metal surfaces," Optical Engineering 35 (3), pp. 845-857, 1996. 